\title{
Anisotropy and scaling corrections in turbulence
}

\author{
Detlef Lohse ${ }^{1, *}$ and Axel Müller-Groeling ${ }^{2}$ \\ ${ }^{1}$ The James Franck Institute, The University of Chicago, 5640 South Ellis Avenue, Chicago, Illinois 60637 \\ and Fachbereich Physik, University of Marburg, Renthof 6, 35032 Marburg, Germany \\ ${ }^{2}$ Department of Physics, University of Toronto, 60 St. George Street, Toronto, Ontario, Canada M5S 1A7 \\ and Max Planck Institute für Kernphysic, Postfach 103980, D-69029 Heidelberg, Germany
}

(Received 19 January 1995; revised manuscript received 29 December 1995)

\begin{abstract}
We analyze second-order turbulent velocity moments both in $r$ and in $p$ space. Finite size corrections induce dramatic differences between local $r$ - and $p$-space scaling exponents. As analytically accessible examples we focus on two popular parametrizations: the Batchelor parametrization for the $r$-space structure function and a common parametrization for the energy spectrum, $E(p) \propto p^{-5 / 3} \exp \left(-p / p_{d}\right)$. The spectral bottleneck energy pileup hidden in the Batchelor parametrization results in an extended $r$-space scaling range, comparable to experimental ones for the same Taylor-Reynolds number $\mathrm{Re}_{\lambda}$. Shear effects are discussed in terms of (global) apparent scaling correction $\delta \zeta^{a p p}\left(\operatorname{Re}_{\lambda}\right)$ to classical scaling, which again depend on whether looked at in $r$ or in $p$ space. The differences can be traced back to the subtleties of the crossovers in the velocity moments. Our observations emphasize the need for more experimental information on crossovers between different subranges. [S1063-651X(96)04006-8]
\end{abstract}

PACS number(s): PACS: 47.27.-i

\section{INTRODUCTION}

In the theory of fully developed turbulence, scaling ranges of velocity moments in $r$ and in $p$ space are often put into a one-to-one correspondence with each other. The two scaling exponents associated with the scaling ranges are believed to be equivalent. While this view is correct for an infinite system (with an infinite scaling range) the relation between $r$-space and $p$-space exponents becomes more complicated for finite Reynolds numbers. It is the aim of this paper to quantitatively examine these finite size effects, as they might well be essential to interpret experiments $[1-8]$ and numerical simulations [9-11].

We first define the infinite scaling range exponents $\zeta_{m}$. In $r$ space, they are defined via the velocity structure functions

$$
D^{(m)}(r)=\left\langle[\boldsymbol{u}(\boldsymbol{x}+\boldsymbol{r})-\boldsymbol{u}(\boldsymbol{x})]^{m}\right\rangle \propto r^{\zeta_{m}}
$$

From a theoretical point of view [12-14], the $p$-space scaling exponents corresponding to the (discrete) Fourier transformation $\boldsymbol{u}(\boldsymbol{p})$ of the velocity field $\boldsymbol{u}(\boldsymbol{x})$ are more easily accessible,

$$
\left\langle|\boldsymbol{u}(\boldsymbol{p})|^{m}\right\rangle \propto p^{-\zeta_{m}}
$$

Kolmogorov's classical dimensional analysis of the turbulence problem [15] gives, of course, the same result for both kinds of scaling exponents, namely, $\zeta_{m}=m / 3$. Since Landau's famous footnote in Ref. [16] it has been a matter of interest whether there are scaling corrections $\delta \zeta_{m}=\zeta_{m}-m / 3$ to the classical result in the limit of infinite Reynolds number Re $[17,18,13,14]$.

Grossmann and Lohse discovered and analyzed finite size scaling corrections in their reduced wave vector set approxi-

\footnotetext{
*Electronic address: lohse@cs.uchicago.edu
}

mations (REWA; see [19-22] and references therein) of the Navier-Stokes equations. The stirring subrange (SSR) scaling corrections at the infrared end of the spectrum are presumably due to the broken symmetry of the Navier-Stokes dynamics because of the finite size of the system: For small $p$ only downscale energy transfer is possible, i.e., the translational invariance and the self-similarity of the turbulent flow is broken by the boundary conditions. In addition to the investigation of these local scaling corrections, REWA also offered the opportunity to study the $\operatorname{Re}_{\lambda}$ dependence of global corrections to classical scaling. Values of $\mathrm{Re}_{\lambda}$ ranging from $10^{2}$ to $10^{4}$ could be simulated $[20,23]$ and it was shown [23] that $\delta \zeta_{m} \propto \operatorname{Re}_{\lambda}{ }^{-3 / 5}$ due to the spectral corrections to classical scaling,

$$
\left\langle|\boldsymbol{u}(\boldsymbol{p})|^{m}\right\rangle \propto p^{-m / 3}\left[1+\alpha_{m}\left(\frac{p}{p_{s}}\right)^{-2 / 3}\right],
$$

which result from large scale anisotropy (e.g., shear). The $p^{-2 / 3}$ shear correction has first been suggested by Lumley [24], who employed dimensional analysis, and was later also found in Refs. [25,23,26,27] with the help of dimensional analysis in terms of Clebsch variables. The parameter $p_{s}$ is the typical scale set by the strength of the shear $s$, $p_{s}=\sqrt{s^{3} / \epsilon}$, and $\alpha_{m}$ a dimensionless parameter, presumably on the order of 1. It is not yet clear whether the second term in Eq. (3) is only a small-p correction or whether pure shear energy spectra $E(p) \propto\left\langle|\boldsymbol{u}(\boldsymbol{p})|^{2}\right\rangle / p \propto p^{-7 / 3}$ exist, i.e., whether $p_{s} \gg p_{L}$ can be achieved. Here, $p_{L} \equiv 1 / L$ is the momentum scale set by the external stirring force. Yakhot [26] recently discussed experimental indications for pure shear spectra. In cross spectra [28] $E_{12}(p)$ they have experimentally been identified by Saddoughi and Veeravalli [6].

A systematic analysis of the properties of $r$ - and $p$-space scaling exponents in finite- $\mathrm{Re}_{\lambda}$ turbulence has been performed by the present authors in Ref. [29]. We demonstrated that the $r$-space crossover from the inertial subrange 
(ISR) to the viscous subrange (VSR) and from the ISR to a large- $r$ saturation range can lead to energy pileups at both ends of the $p$-space ISR, the so-called bottleneck phenomenon [30]. In other words, monotonous local $r$-space scaling exponents may give rise to nonmonotonous local $p$-space exponents. Both physical (based on the conserved energy current in $p$ space) and formal explanations for this effect as well as a comparison with available experimental and numerical data were given in Ref. [29].

In the present paper, we continue and extend our investigation of finite size effects on local scaling exponents in $r$ and in $p$ space. For the time being, analytical techniques that would enable us to study these questions directly from a dynamical point of view are not available. Therefore we have to adopt a more phenomenological approach where measured or (numerically) calculated velocity structure functions are described by certain parametrizations. These parametrizations can be formulated either in $r$ or in $p$ space and their quality can be checked by direct comparison with experimental or numerical data. As a central point in our paper we exploit the fact that any description of a structure function in $r$ or in $p$ space implies at the same time a certain statement in the respective Fourier spaces. It is therefore legitimate and, as we believe, even necessary to test a given parametrization against all available information in both $r$ and $p$ spaces. A similar approach for energy dissipation correlations was followed by Nelkin [31].

Our central finding is that the scaling quality of the same parametrization - once looked at in $r$ space, once looked at in $p$ space - is rather different. This dramatic difference is not an artefact of the chosen parametrization but a mathematical property of the Fourier transformation. Once the parametrization (as a "best fit" to the data) is accepted in $r(p)$ space, its corresponding Fourier transform to $p(r)$ space has to be accepted as well - in spite of the often surprising features.

We restrict ourselves to the two probably most common parametrizations (and finite size corrections thereof) for the scaling behavior of velocity moments, namely, the $p$-space parametrization (4) discussed by Foias, Manely, and Sirovich [32] (henceforth called FMS parametrization for simplicity; see also Frisch et al. [33]) and the Batchelor parametrization (5) $[34,28,35]$, common in $r$ space. The latter has been very common in particular in the engineering literature. Recently Sirovich, Smith, and Yakhot [36] have given some interesting theoretical foundation for it. We will see that the main difference between FMS and Batchelor parametrization lies in the description of the crossover from the viscous to the inertial range. Pointing out the importance and the surprising consequences of the nature of the crossovers is a main aspect of our work, since up to now research was mainly concerned with infinite Re scaling exponents.

The restriction to the parametrizations is only for technical conveniance. Our main result, Fig. 4, which reveals the differences in scaling quality in $r$ vs $p$ space, can be understood without referring to any parametrization. These differences appear to be somewhat less pronounced (but not absent) in experimental data. The reason presumably lies in the experimental necessity to perform some sort of running average to reduce the noise in the data. In experiments it is therefore always dealt with locally averaged scaling expo- nents as opposed to the strictly local ones considered in this paper. Indeed, we show that bottleneck energy pileups [29] [i.e., a nonmonotonous local scaling exponent $\zeta(p)$ ] hidden in Batchelor's $r$ space parametrization considerably reduces when $p$ spectra are locally averaged as has to be done for experimental data.

The paper is organized as follows: Sections II and III deal with local small- $p$ scaling corrections. The bottleneck energy pileup at the infrared end of the ISR [29] as well as the small- $p$ scaling corrections found in REWA [19] and also by dimensional analysis in terms of Clebsch variables $[23,25,26]$ belong to this category. We believe that these are all manifestations of the broken Navier-Stokes symmetry due to the boundaries, i.e., large scale anisotropy [19]. To investigate possible relations among these effects we modify the above-mentioned parametrizations to describe the scaling corrections from REWA, examine the ensuing consequences in $r$ space, and perform a quantitative comparison for the local scaling exponents $\zeta_{2}(p)$ resulting from the three different approaches in Refs. [19,23,29].

In Sec. IV we focus our attention on the Taylor-Reynolds number dependences of the apparent (global) scaling corrections $\delta \zeta_{2}^{a p p}$ due to shear effects. While it is probably not too surprising that Batchelor and FMS parametrization lead to different $\operatorname{Re}_{\lambda}$ dependences of the apparent scaling corrections $\delta \zeta_{2}^{a p p}$, our result that both parametrizations give rise to different behavior of $\delta \zeta_{2}^{a p p}$ in momentum and coordinate space, respectively, is certainly unexpected. E.g., we find that the $p$-space result $\delta \zeta_{2}^{a p p, p} \propto \operatorname{Re}_{\lambda}{ }^{-3 / 5}$ of Refs. [23,13] corresponds to $\delta \zeta_{2}^{a p p, r} \propto \operatorname{Re}_{\lambda}{ }^{-1 / 2}$ in $r$ space. This observation can be viewed as yet more evidence for the fact that finite size effects can render $r$ - and $p$-space exponents inequivalent. Section V is left to a summary and to conclusions.

\section{BATCHELOR VERSUS FMS PARAMETRIZATION}

\section{A. Definitions and Fourier transforms}

To describe the behavior of energy spectra $E(p)$, the FMS parametrization [32,33]

$$
E_{F M S}(p)=E_{0} \epsilon^{2 / 3} p^{-5 / 3} \exp \left(-p / p_{d}\right)
$$

has frequently been used to interpret experimental $[37,38]$ and numerical $[10,19,20]$ data. Here, $E_{0}$ is the $p$-space Kolmogorov constant, $\epsilon$ the energy dissipation rate, and $p_{d}$ characterizes the crossover to the viscous range. On the other hand, measured structure functions $D^{(2)}(r)$ are well described by the Batchelor parametrization $[34,28,36,1,35,39,29,40]$,

$$
D_{B}^{(2)}(r)=\frac{\epsilon r^{2} /(3 \nu)}{\left[1+\left(\frac{1}{3 b}\right)^{3 / 2}\left(\frac{r}{\eta}\right)^{2}\right]^{2 / 3}},
$$

where $\nu$ is the viscosity, $\eta=\nu^{3 / 4} / \epsilon^{1 / 4}$ the Kolmogorov length, and $b=27 \Gamma(4 / 3) E_{0} / 5=6.0-8.4$ the experimentally determined $[28,41] r$-space Kolmogorov constant. The generalization of both parametrizations to $\zeta_{2} \neq 2 / 3$ is straightforward [40] and was considered in [29]. The essential aspects of our present work do not depend on the precise value of $\zeta_{2}$. Clearly, both parametrizations neglect the finite size of 
the system since they do not contain a scale for the external stirring force. Furthermore, the FMS-parametrization equation (4) does not contain any energy pileup (or bottleneck effect) $[30,29]$. The velocity structure function for a given energy spectrum can be calculated through the Fourier transformation [28]

$$
D^{(2)}(r)=4 \int_{0}^{\infty} E(p)\left(1-\frac{\sin (p r)}{p r}\right) d p .
$$

Inverting this equation, i.e., calculating the energy spectrum from a given structure function, requires a short discussion. Let us consider turbulence in a large but finite domain, so that $E(p) \rightarrow 0$ as $p \rightarrow 0$. Then the term involving no trigonometric function on the right-hand side (rhs) of Eq. (6) is finite, $4 \int_{0}^{\infty} E(p) d p \equiv D^{(2)}(\infty)<\infty$. Physically, this term corresponds to the total energy in the fluid. We can now straightforwardly invert Eq. (6) to give

$$
E(p)=-\frac{1}{2 \pi} \int_{0}^{\infty} p r \sin (p r)\left[D^{(2)}(r)-D^{(2)}(\infty)\right] d r
$$

In the limit of infinite system size $D^{(2)}(\infty)$ grows beyond all bounds, rendering Eq. (7) ill-defined at first sight. However, since $\int_{0}^{\infty} p r \sin (p r) d r \propto p \delta^{\prime}(p)$, this affects only singular contributions at the origin which we may safely discard. We will therefore always use Eq. (7) with the understanding that $D^{(2)}(\infty)=0$. Formally this means nothing more but that the Fourier transformation of a function will not change (apart from the $\delta$ function) if the function is shifted by a constant. With the help of the transformation equations (6) and (7) we can calculate the structure function corresponding to the FMS parametrization (4) and the energy spectrum associated with the Batchelor parametrization (5), giving

$$
\begin{aligned}
D_{F M S}^{(2)}(r)= & \frac{4 E_{0} \Gamma(-2 / 3)}{r(5 / 3) p_{d}^{5 / 3}}\left\{\frac{5}{3} p_{d} r-\left(1+p_{d}^{2} r^{2}\right)^{5 / 6}\right. \\
& \left.\times \sin \left[\frac{5}{3} \arctan \left(p_{d} r\right)\right]\right\}
\end{aligned}
$$

and

$$
\begin{aligned}
E_{B}(p) & =-\frac{1}{4 \pi} \frac{\epsilon}{3 \nu} p r_{d}^{\prime} \frac{d^{3}}{d p^{3}} \int_{-\infty}^{\infty} \frac{\exp \left(i p r_{d}^{\prime} x\right)}{\left(1+x^{2}\right)^{2 / 3}} d x \\
& =E_{0} \epsilon^{2 / 3} p_{d}^{\prime-5 / 3} A\left[\frac{2}{3} \widetilde{p}^{1 / 6} K_{11 / 6}(\widetilde{p})+\widetilde{p}^{-1 / 6} K_{5 / 6}(\widetilde{p})\right],
\end{aligned}
$$

respectively. Here, we introduced some abbreviations for simplicity: The dimensionless constant $A$ has the value $A=[9 \Gamma(1 / 3)] /\left[\sqrt{2 \pi} 2^{2 / 3} 5 \Gamma(2 / 3)\right], \tilde{p}=p r_{d}^{\prime}$, and $K_{\nu}$ is the modified Bessel function of the third kind [42]. A result analogous to our Eq. (9) has been earlier obtained by Sirovich, Smith, and Yakhot [36]. These authors transformed the Batchelor parametrization for the longitudinal structure function to $p$ space. [Whether $D(r)$, the longitudinal structure function $D_{L}(r)$, or the transversal one $D_{T}(r)$ is best approximated by a Batchelor parametrization has to be decided by comparison with experimental data. Clearly, if one chooses a

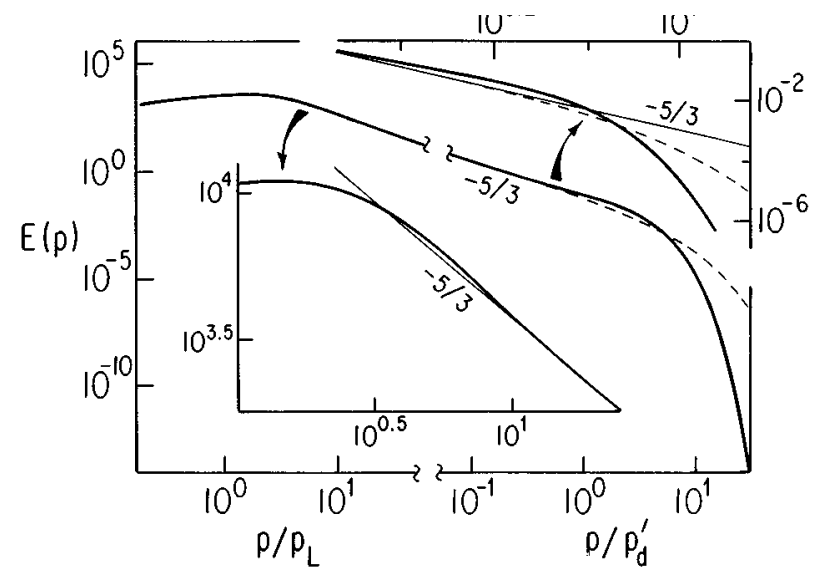

FIG. 1. Energy spectrum equation (9) (solid) with the energy pileup, and the spectrum equation (4) (dashed) without it. We assumed a Batchelor-type infrared (IR) cutoff, Eq. (15). The two arrows point to blowups of the spectrum in the crossover regions. In these enlargements it is compared to classical $-5 / 3$ scaling.

certain parametrization for one of them, there is no freedom left for the others (assuming isotropy).] In Ref. [29] the bottleneck effect (see also Sec. II B) and its important physical consequences hidden in the transformed Batchelor formula were discussed for the first time.

The crossover from the inertial to the viscous range is characterized by $r_{d}, p_{d}$ for the FMS parametrization and by $r_{d}^{\prime}, p_{d}^{\prime}$ for the Batchelor parametrization. The large- and small- $r$ limits of $D^{(2)}(r)$ are required to give $D^{(2)}(r)=b(\epsilon r)^{(2 / 3)}$ and $D^{(2)}(r)=\epsilon r^{2} /(3 \nu)$, respectively. Equation (5) is obviously designed to meet these constraints and comparing the asymptotic relations with the appropriate limits of Eq. (8) fixes $E_{0}=5 b[\Gamma(4 / 3)]^{-1} / 27=1.74$ and

$$
p_{d}^{-1}=(10 b / 27)^{3 / 4} \eta \approx 2.34 \eta
$$

where we chose $b=8.4$ [28]. Now, $r_{d}$ and $r_{d}^{\prime}$ are defined by equating the asymptotic limits, $\epsilon r^{2} /(3 \nu)=b(\epsilon r)^{2 / 3}$, so that we arrive at

$$
r_{d}=r_{d}^{\prime}=(3 b)^{3 / 4} \eta \approx 11.25 \eta
$$

We note, however, that although $r_{d}$ and $r_{d}^{\prime}$ are the same (by definition), $D_{B}^{(2)}(r)$ shows a sharper crossover from VSR to ISR than $D_{F M S}^{(2)}(r)$, i.e., $D_{B}^{(2)}(r) \geqslant D_{F M S}^{(2)}(r)$ for all $r$. This can be seen in Fig. 1 of Ref. [29] where we compared $D_{B}^{(2)}(r)$ with $D_{F M S}^{(2)}(r)$. Finally, the $p$-space crossovers $p_{d}$ and $p_{d}$, are defined by the cutoff in the exponential decay of the spectrum for large $p$. Thus, $p_{d}$ is determined by Eq. (4) and, since $K_{\nu}\left(\tilde{p}=p r_{d}{ }^{\prime}\right) \propto p^{-1 / 3} \exp \left(-p r_{d}^{\prime}\right)$ for large argument, we have

$$
p_{d}^{\prime-1}=r_{d}^{\prime} \approx 11.25 \eta
$$

Note that the naive expectation that ( $p$-space crossover) $\times$ ( $r$-space crossover $) \approx 2 \pi$, holds in neither case. For Eqs. (4) and (8) we have $r_{d} p_{d} \approx 4.8$ whereas for Eqs. (5) and (9) we have simply $r_{d}^{\prime} p_{d}^{\prime}=1$. 


\section{B. Bottleneck phenomenon}

In [29] we showed that in contrast to Eq. (4) the parametrization Eq. (5) contains an important physical phenomenon, the bottleneck effect [30]. This becomes apparent when comparing the energy spectra $E_{F M S}(p)$ and $E_{B}(p)$ in Fig. 1. For small $p$ both functions coincide. Around $p \approx p_{d}^{\prime}$, however, an energy pileup in the crossover region of $E_{B}(p)$ becomes noticeable, leading to a nonmonotonous logarithmic slope $d \ln E_{B} / d \ln p$. The local $r$ - and $p$-space scaling exponents

$$
\zeta_{2}(r)=\frac{d \ln D^{(2)}(r)}{d \ln r}, \quad-\zeta_{2}(p)-1=\frac{d \ln E(p)}{d \ln p}
$$

of both the Batchelor and the FMS parametrization are plotted in the right part of Fig. 2. The minimal local $p$-space scaling exponent of the spectrum $E_{B}(p)$ is 0.44 , i.e., the scaling correction is an order of magnitude larger than the discussed intermittency corrections $[28,17]$. This effect could explain that the spectra in numerical simulations $[9,10,43]$ are flatter than the classical expectation, rather than being steeper as one might expect from possible intermittency corrections.

Furthermore, in Ref. [29] we considered a straightforward generalization of the Batchelor parametrization,

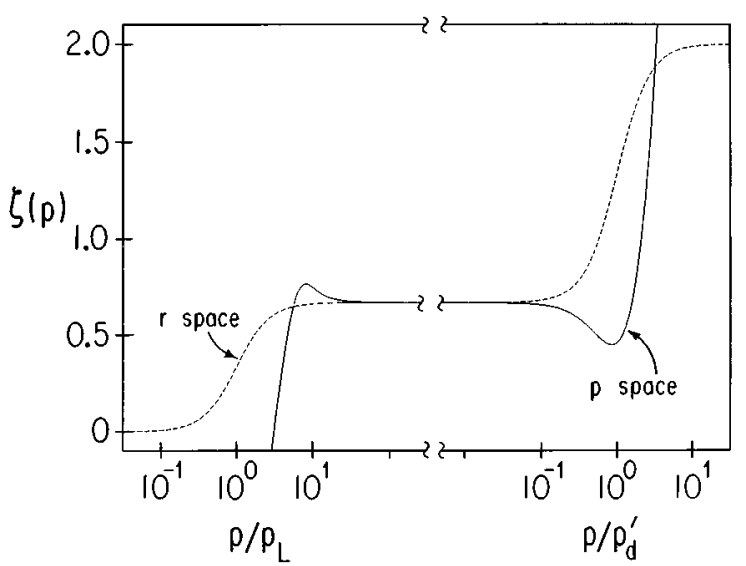

FIG. 2. The local $p$-space scaling exponents $\zeta(p)$ (solid), and the local $r$-space scaling exponent $\zeta(r=1 / p)$.

$$
D_{B}^{(2)}(r) \propto r^{2}\left(r_{d}^{\prime 2}+r^{2}\right)^{-2 / 3}\left(L^{2}+r^{2}\right)^{1 / 3},
$$

which accounts for the crossover from the ISR to a large- $r$ saturation range induced by the finite scale $L=1 / p_{L}$ set by the external stirring force. This second crossover might well not be universal, but our parametrization agrees reasonably well with available data $[1,4,28,39]$. Calculating the corresponding spectrum we obtained for $r_{d}^{\prime} \ll r$

$$
\begin{aligned}
E_{B}(p)= & \frac{\left\langle\mathbf{u}^{2}\right\rangle L}{\pi}\left\{-\frac{\Gamma(5 / 6)}{\Gamma(1 / 3)} \sqrt{\pi}\left[\frac{5}{9} \bar{p}^{2}{ }_{1} F_{2}\left(\frac{11}{6}, \frac{5}{2}, \frac{5}{2}, \frac{\bar{p}^{2}}{4}\right)+\frac{11}{405} \bar{p}_{1}^{4} F_{2}\left(\frac{17}{6}, \frac{7}{2}, \frac{7}{2}, \frac{\bar{p}^{2}}{4}\right)\right]\right. \\
& \left.+\frac{\pi}{2}\left[\frac{1}{3} \bar{p}_{1} F_{2}\left(\frac{4}{3}, 2, \frac{3}{2}, \frac{\bar{p}^{2}}{4}\right)+\frac{2}{27} \bar{p}_{1}^{3} F_{2}\left(\frac{7}{3}, 3, \frac{5}{2}, \frac{\bar{p}^{2}}{4}\right)\right]\right\},
\end{aligned}
$$

where $\bar{p}=p / p_{L}$ and ${ }_{1} F_{2}(a, b, c, z)$ denotes a generalized hypergeometric function [44]. The most prominent feature of this expression is a second bottleneck pileup at the infrared end of the $p$-space ISR. The local logarithmic slopes of (14) and (15) are plotted in the left part of Fig. 2. Both bottlenecks have the same physical origin, namely, the broken symmetry due to finite size effects. The symmetry breaking scale is introduced by the stirring force and the finite size of the vessel, wind channel, or atmosphere $[1,3]$ at the infrared end of the spectrum, and by viscosity at the large- $p$ end of the ISR. Formally, both bottleneck energy pileups originate from the sharp $r$-space crossovers defined by the Batchelorparametrization equation (14). The physical explanation $[20,29]$ builds on the constant energy flux $T(p) \sim p u(\boldsymbol{p}) \int d p_{1} d p_{2} u\left(\boldsymbol{p}_{1}\right) u\left(\boldsymbol{p}_{2}\right) \delta\left(\boldsymbol{p}+\boldsymbol{p}_{1}+\boldsymbol{p}_{2}\right) \quad$ downscale in $p$ space. For a detailed discussion of the bottleneck effect we refer to Ref. [29].

\section{Comparision with experiment}

The ultraviolet bottleneck pileup is rather universal and has meanwhile been observed in many experiments and numerical simulations $[9,45]$. The infrared bottleneck clearly is not universal. In the zero-pressure gradient boundary layer experiments of Saddoughi and Veeravalli [6] it shows up for velocity measurements in the outer part of the boundary layer. However, the bottelneck pileup on the low-wavenumber end of the inertial subrange accentuates in the outer part of the layer for the adverse pressure gradient case of Saddoughi [7], where the ratio of the extra strain rate to the basic shear is large. In Figs. 3 we show compensated spectra $E(p) p^{5 / 3}$, which is a common representation of experimental data: In Fig. 3(d) we have plotted the compensated Batchelor spectrum (2.6) and (2.12), and in Figs. 3(a)-(c) the measured ones of Saddoughi and Veeravalli [6] for comparison. The Batchelor parametrization which fits $r$-space data very well $[39,29,35]$ seemingly overestimates the strength of the bottleneck energy pileups.

However, a point to be kept in mind when comparing the strength of the bottlenecks with experimental data is the issue of averaging. While experimental scaling exponents necessarily represent data averaged over a certain interval, we defined a pointwise (local) slope in (13). To estimate the effect of averaging, we performed a running average of the pointwise slopes in Fig. 3(d) using an averaging range of $[p / \sqrt{10}, p \sqrt{10}]$, as done in REWA $[19,20]$. As expected, the bottleneck pileups become attenuated to about half their size and are now quantitatively closer to the measured ones. The 

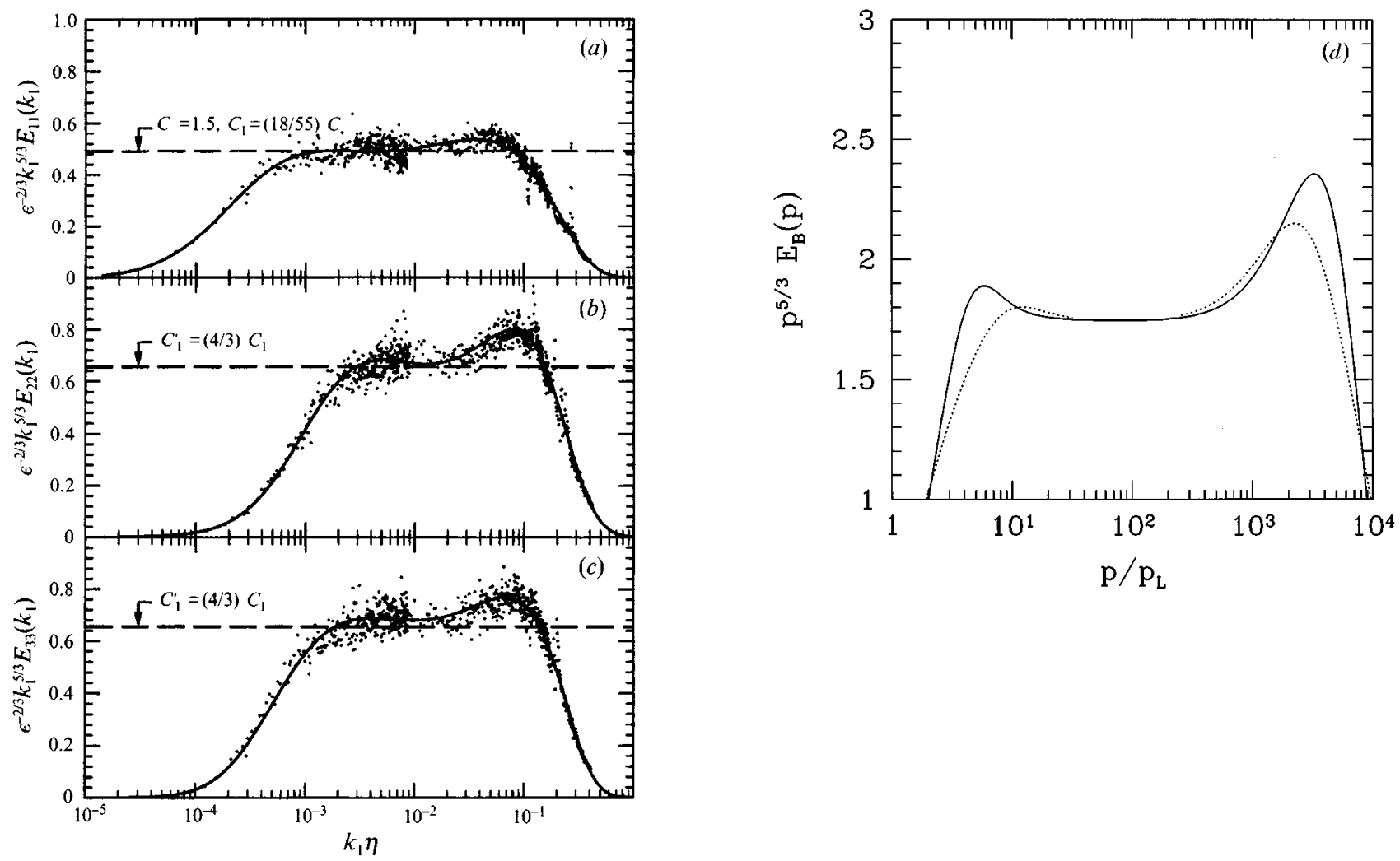

FIG. 3. Compensated (i.e., spectra multiplied by the wave vector $k^{5 / 3}$ ) longitudinal and transversal spectra for $\operatorname{Re}_{\lambda}=1450$ in some boundary layer, as measured by Saddoughi and Veeravalli [6]. (a), (b), and (c) show the $u_{1}, u_{2}$, and $u_{3}$ spectra, respectively. Energy pileups are seen at both ends of the ISR. From the straight line the Kolmogorov constant is estimated, which is not relevant for our context here. (a)-(c) are copied from Ref. [6] with kind permission of the authors. We refer to [6] for more details on the measurements. (d) shows the compensated Batchelor spectrum (2.6) and (2.12) for comparison (solid line). The dashed line shows the averaged (over the range $[p / \sqrt{10}, p \sqrt{10}])$ slope which should be compared with the experimental data.

matter of averaging becomes even more relevant on large scales with its poor statistics. This may be another reason (besides the lack of universality) that the infrared bottleneck is rarely observed.

\section{Higher-order moments}

The preceding analysis cannot easily be extended to higher-order structure functions. The connection between, say, the fourth-order structure function $D^{(4)}(r)$ and the corresponding fourth moment of $\boldsymbol{u}(\boldsymbol{p})$,

$$
\begin{aligned}
D^{(4)}(r) \propto & \int \delta\left(\boldsymbol{p}_{1}+\boldsymbol{p}_{2}+\boldsymbol{p}_{3}+\boldsymbol{p}_{3}\right)\left\langle\boldsymbol{u}\left(\boldsymbol{p}_{1}\right) \boldsymbol{u}\left(\boldsymbol{p}_{2}\right) \boldsymbol{u}\left(\boldsymbol{p}_{3}\right) \boldsymbol{u}\left(\boldsymbol{p}_{4}\right)\right\rangle \\
& \times\left\langle\prod_{j=1}^{4}\left[\exp \left(i \boldsymbol{p}_{j} \cdot \boldsymbol{r}\right)-1\right]\right\rangle_{\text {angle }} d \boldsymbol{p}_{1} d \boldsymbol{p}_{2} d \boldsymbol{p}_{3} d \boldsymbol{p}_{4},
\end{aligned}
$$

is considerably more complicated than Eq. (6). Therefore we have to restrict ourselves to a few general remarks.

Neglecting intermittency corrections we assume as a first approximation that $\boldsymbol{v}_{r}(\boldsymbol{x}, t)=\boldsymbol{u}(\boldsymbol{x}+\boldsymbol{r}, t)-\boldsymbol{u}(\boldsymbol{x}, t)$ and $\boldsymbol{u}(\boldsymbol{p}, t)$ are Gaussian distributed, so that we may simply factorize higher moments (for even $m$ ),

$$
\begin{gathered}
\left\langle|\boldsymbol{u}(\boldsymbol{p})|^{m}\right\rangle \propto\left\langle\left\langle\left.\boldsymbol{u}(\boldsymbol{p})\right|^{2}\right\rangle^{m / 2},\right. \\
D^{(m)}(r) \propto\left[D^{(2)}(r)\right]^{m / 2} .
\end{gathered}
$$

The above assumptions are not independent. In a completely homogeneous medium the second moments in $p$ space are local [i.e., $\left\langle\boldsymbol{u}^{*}(\boldsymbol{p}) \boldsymbol{u}\left(\boldsymbol{p}^{\prime}\right)\right\rangle \propto \delta\left(\boldsymbol{p}-\boldsymbol{p}^{\prime}\right)$ ] and the second line in Eq. (17) is a direct consequence of the first one. Of course, the assumption of Gaussian factorization in Eq. (17) is at variance with the fact that odd moments do not vanish [e.g., $D^{(3)}(r)<0$ for large $r$ due to Kolmogorov's structure equation [28]].

The similar looking factorizations in Eq. (17) lead to quite different results concerning the $m$ dependence of the crossovers $r_{d}^{\prime(m)}$ and $p_{d}^{(m)}$ (or $p_{d}^{\prime(m)}$ ) between VSR and ISR. These lengths are defined as above by matching the asymptotic behavior for large and for small $r$ and by the cutoff in the exponentials, respectively. In $r$ space we get

$$
r_{d}^{\prime(m)}=r_{d}^{\prime(2)}=r_{d}^{\prime}=\text { const }
$$

for all $m$, which is in agreement with recent measurements [40], while in $p$ space

$$
p_{d}^{(m)}=2 p_{d}^{(2)} / m=2 p_{d} / m
$$


becomes smaller with increasing $m$. (The same relation holds for $p_{d}^{\prime}$.) Equation (19) has been numerically confirmed to a high precision [19]. Thus, for increasing $m$ the ISR becomes smaller and smaller in $p$ space, whereas it remains invariant in $r$ space. Technically, this is due to the fact that we compare two power laws in $r$ space and a power law with an exponential in $p$ space. An intuitive understanding is provided by the following remark: Raising $D^{(2)}(r)$ to some power smoothes the transition from VSR to ISR and consequently reduces the corresponding spectral strength at large values of $p$.

\section{SMALL-p SCALING CORRECTIONS}

\section{A. Infrared cutoff}

In order to define a finite range for the infrared scaling corrections discussed below we introduce the external stirring force scale $L=p_{L}^{-1}$ into the $p$-space parametrizations $E_{B}(p)$ and $E_{\mathrm{FMS}}(p)$ : Both spectra are multiplied by $(2 / \pi) \arctan \left[\left(p / p_{L}\right)^{11 / 3}\right]$. This amounts to imposing energy equipartition $E(p) \sim p^{2}$ on the unforced wave vector modes $[28,46,18]$ with $p \ll p_{L}$. The corresponding structure functions saturate for $r>L, D^{(2)}(r>L) \approx D^{(2)}(\infty)=$ const.

We can now introduce the Taylor-Reynolds number $\operatorname{Re}_{\lambda}=\lambda u_{1, r m s} / \nu$, where $\lambda=u_{1, r m s} /\left(\partial_{1} u_{1}\right)_{r m s}$ is the Taylor length and $\nu$ the viscosity. To express $p_{d}$ and $p_{d}^{\prime}$ [see (10) and (12)] in terms of $L$ and $\operatorname{Re}_{\lambda}$ we note that $\epsilon=c_{\epsilon} u_{1, r m s}^{3} / L$ with $c_{\epsilon} \approx 1$ (from grid turbulence experiments [47]). We neglect the $\operatorname{Re}_{\lambda}$ dependence of $c_{\epsilon}$ for small $\operatorname{Re}_{\lambda}$ [48]. On the other hand, $\epsilon=15 \nu\left(\partial_{1} u_{1}\right)_{r m s}^{2}$ [28] and we finally get $\eta=15^{3 / 4} c_{\epsilon}^{-1} L \operatorname{Re}_{\lambda}^{-3 / 2}$. With $c_{\epsilon} \approx 1$ and using (10) and (12) this means

$$
\begin{aligned}
& p_{d}^{-1} \approx 18 L \operatorname{Re}_{\lambda}^{-3 / 2}, \\
& p_{d}^{\prime-1} \approx 86 L \operatorname{Re}_{\lambda}^{-3 / 2},
\end{aligned}
$$

so that we have connected the length scales $r_{d}\left(\equiv r_{d}^{\prime}\right)$ and $L$ with the Taylor-Reynolds number $\operatorname{Re}_{\lambda}$.

\section{B. REWA scaling corrections and structure functions}

Motivated by deviations $\delta \zeta_{m}(p)$ from classical scaling found by Grossmann and Lohse [19] in the SSR (and, also, in the VSR) we model a spectrum according to the numerical results in [19] and numerically Fourier transform it into $r$ space. We focus attention on $p$-space SSR scaling corrections alone. The scaling corrections are introduced as follows: For any spectrum $E(p)$ with $d \ln E / d \ln p=-\zeta(p)-1$ a modification defined by

$$
\widetilde{E}(p)=E(p) \frac{\left(p_{b}^{\beta}+p^{\beta}\right)^{\delta / \beta}}{p^{\delta}}
$$

leads to a local exponent

$$
\widetilde{\zeta}(p)=\zeta(p)+\frac{\delta}{1+\left(p / p_{b}\right)^{\beta}} .
$$

The three parameters $\delta, \beta, p_{b}$ determine size [i.e., $\widetilde{\zeta}(p)=2 / 3+\delta$ for small $p]$, smoothness and onset of the in-
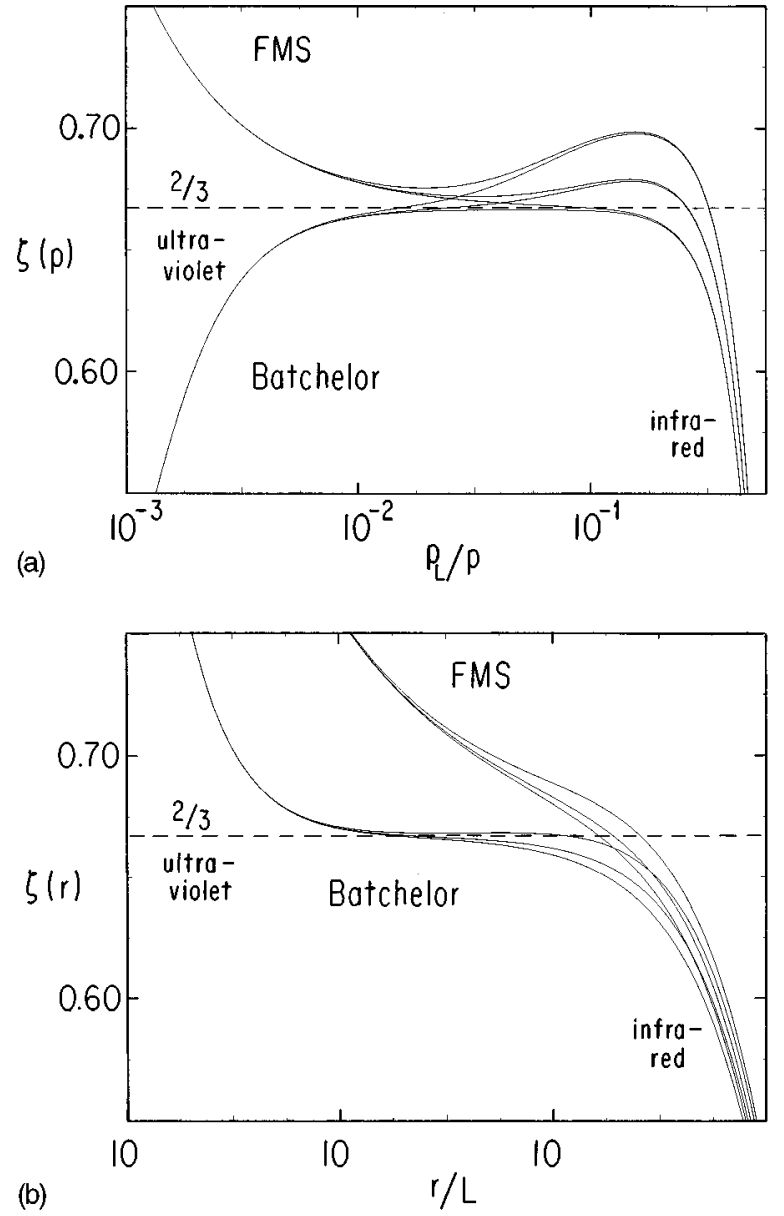

FIG. 4. (a) Local $p$-space scaling exponents $\zeta(p)$ of the FMS and the Batchelor-type energy spectra, both with the arctan cutoff for small $p$, see text. To allow for comparison with $r$ space (b), we plotted $\zeta(p)$ versus $p_{L} / p$ rather than versus $p / p_{L}$. The Taylor Reynolds number is $\operatorname{Re}_{\lambda}=3000$; cf. Eqs. (20),(21). From bottom to top on the rhs of the figure, the three pairs of curves correspond to (i) no small- $p$ scaling corrections, (ii) small- $p$ scaling corrections according to (24) with $\delta=0.02$ and $p_{b} / p_{L}=10$, and (iii) small- $p$ scaling corrections with $\delta=0.04, p_{b} / p_{L}=15$. Note that on the scale of this plot the bottleneck on the left hand side of the figure is quite large and only partly seen. (b) Local $r$-space scaling exponents $\zeta(r)$ for the six curves of (a). From bottom to top for both the FMS and Batchelor triple of curves: no scaling corrections, $\delta=0.02$, $p_{b} / p_{L}=10$ and $\delta=0.04, p_{b} / p_{L}=15$.

frared scaling corrections, respectively. We choose $\delta=0.00-0.04, \beta=2$, and $p_{b} \approx 10 p_{L}-15 p_{L}$ as suggested by the numerical findings in [19]. The two spectra

$$
\begin{gathered}
\widetilde{E}_{F M S}(p)=E_{F M S}(p) \frac{\left(p_{b}^{2}+p^{2}\right)^{\delta / 2}}{p^{\delta}}, \\
\widetilde{E}_{B}(p)=E_{B}(p) \frac{\left(p_{b}^{2}+p^{2}\right)^{\delta / 2}}{p^{\delta}}
\end{gathered}
$$

were each multiplied by the small $p$ cutoff $(2 / \pi) \arctan \left[\left(p / p_{L}\right)^{11 / 3+\delta}\right]$ and then numerically Fourier transformed. The local scaling exponents for $p$ and $r$ space [defined as in (13)] are shown in Figs. 4(a) and 4(b), respectively. We chose $\operatorname{Re}_{\lambda}=3000$ as in typical experiments [1]. 
For the FMS parametrization without scaling corrections ( $\delta=0$ ) a well-defined scaling range in $p$ space (about one decade) corresponds to very bad scaling behavior in $r$ space. REWA spectra are well described by the FMS parametrization $[19,20,22]$ and consequently the corresponding local exponent $\zeta(r)$ in [22] looks very similar to our present result. The scaling properties of $D_{\mathrm{FMS}}(r)$ is worse than that for the experimental structure function for the same $\operatorname{Re}_{\lambda}=3000$ [1]. Introducing finite small- $p$ scaling corrections ( $\delta=0.02,0.04$ ) leads to improved (but still poor) $r$-space scaling, while the $p$-space exponent becomes nonmonotonous by construction.

The Batchelor parametrization exhibits much better scaling properties to begin with, even for $\delta=0$. The influence of the infrared cutoff can be partly compensated by small $p$ scaling corrections (again $\delta=0.02,0.04$ ). The ensuing $r$-space behavior is then quite comparable with the experimentally realized scaling ranges of about 1.5-2 decades for $\operatorname{Re}_{\lambda}=3000$ [1].

To summarize: Our findings concerning small- $p$ scaling corrections support and reinforce the conclusion drawn earlier when investigating the bottleneck phenomena. The simple arctan or exponential cutoffs of the $p$-space ISR scaling range lead to unrealistically short $r$-space scaling ranges. Only the energy pileups at both ends of the $p$-space ISR lead to a realistic scaling range of the structure function, if compared to experiment [1]. Batchelor's parametrizations (2.11), (2.12) of the crossovers include these energy pileups and give a realistic scaling range for given $\mathrm{Re}_{\lambda}$. Our findings also explain why the $r$-space scaling found in REWA is worse than that for the $p$-spectra [19], as the latter is quite well described by the FMS parametrization [22]. It is hard to see how realistic scaling ranges in $r$ space can come about in the absence of $p$-space bumps at both ends of the ISR.

\section{SHEAR EFFECTS}

\section{A. Shear parametrizations}

The generalized FMS parametrization [cf. Eqs. (3) and (4)], which includes the effects of shear,

$$
\left\langle|\boldsymbol{u}(\boldsymbol{p})|^{m}\right\rangle \propto p^{-m / 3}\left[1+\alpha_{m}\left(\frac{p}{p_{L}}\right)^{-2 / 3}\right] \exp \left(-p / p_{d}\right),
$$

was shown [23] to lead to an apparent scaling correction defined by

$$
\delta \zeta_{m}^{a p p, p}=\min _{p}\left[\zeta_{m}(p)\right]-m / 3 .
$$

As usual, $\zeta_{m}(p)=-d \ln \left\langle|\boldsymbol{u}(\boldsymbol{p})|^{m}\right\rangle / d \ln p$. For $p_{L} \ll p_{d}$ it was found that

$$
\begin{aligned}
\delta \zeta_{m}^{a p p, p} & =\operatorname{sgn}\left(\alpha_{m}\right) \frac{10}{9}\left(\frac{9 m p_{L}}{8 p_{d}}\right)^{2 / 5} \alpha_{m}^{3 / 5}=c_{m} \operatorname{Re}^{-3 / 10} \\
& =c_{m}^{\prime} \operatorname{Re}_{\lambda}^{-3 / 5}
\end{aligned}
$$

The apparent scaling corrections vanish with increasing $\operatorname{Re}_{\lambda}$ with a $-3 / 5$ power law for all $m$. From now on we will only consider the case $m=2$. The local scaling exponent following from (25) is shown in Fig. 5(a) for $\operatorname{Re}_{\lambda}=3000$ and

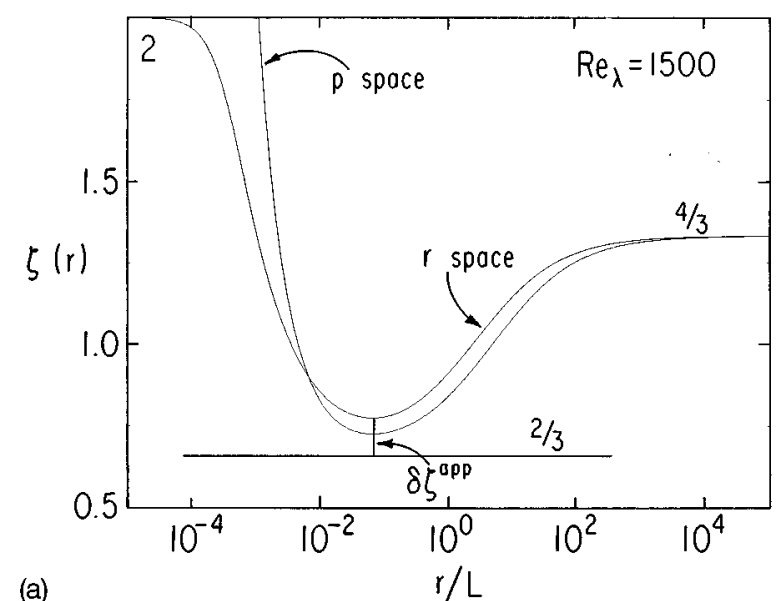

(a)

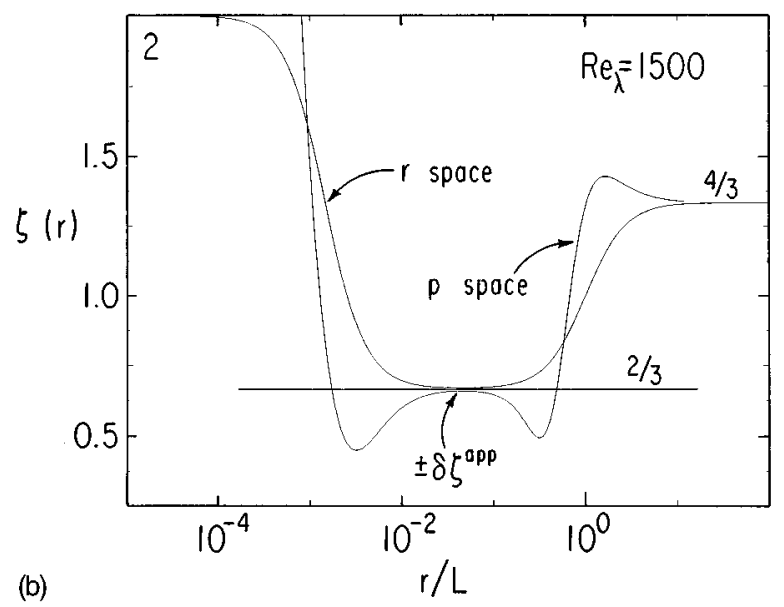

FIG. 5. (a) Local scaling exponents $\zeta(r)$ and $\zeta(p=\gamma / r)$ when shear corrections according to (25) are present, with $\operatorname{Re}_{\lambda}=1500$ $\left(p_{d}=3227\right)$ and $\alpha_{2}=1$. The parameter $\gamma$ serves to shift the $p$-space curve slightly to ensure that the minima of the two curves coincide. (b) As in (a), but now shear corrections according to (28) and its Fourier transform. Again, we chose $\operatorname{Re}_{\lambda}=1500$ $\left(p_{d}^{\prime}=r_{d}^{-1}=676\right)$.

$\alpha_{2}=1 . \operatorname{Re}_{\lambda}$ has been determined using (20). The correction $\delta \zeta_{2}^{a p p, p} \approx 0.06$ is comparatively large but depends through the factor $\alpha_{2}^{3 / 5}$ on our choice of $\alpha_{2}$. The $\operatorname{Re}_{\lambda}$ dependence of $\delta \zeta_{2}^{a p p, p}$ is displayed in Fig. 6 and for large $\mathrm{Re}_{\lambda}$ the asymptotic result (27) is recovered.

The form of the shear correction in (25) is based on nothing but dimensional analysis. Applying the same reasoning directly to the Batchelor parametrization in $r$ space and assuming the shear transition to be of the Batchelor type we are led to the ansatz

$$
D_{B}(r)=\frac{\epsilon}{3 \nu} r^{2} \frac{r_{d}^{\prime 4 / 3}}{\left(r_{d}^{\prime 2}+r^{2}\right)^{2 / 3}} \frac{r_{s}^{-2 / 3}}{\left(r_{s}^{2}+r^{2}\right)^{-1 / 3}}
$$

with $r_{s}=p_{s}^{-1}=\sqrt{\epsilon / s^{3}}$. We assume that shear sets in at the stirring scale, hence $r_{s}=L$. Equation (28) is a generalization of the Batchelor parametrization in complete analogy to the generalized FMS parametrization (25). The former is as le- 


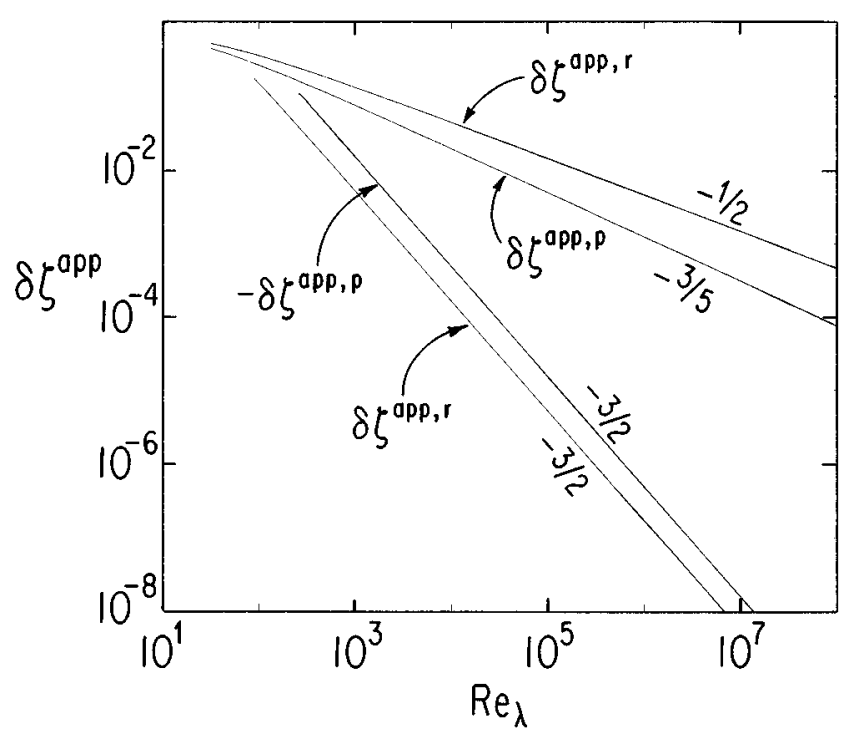

FIG. 6. Double-logarithmic plot of $\delta \zeta^{a p p, r}\left(\operatorname{Re}_{\lambda}\right)$ and $\delta \zeta^{a p p, p}\left(\operatorname{Re}_{\lambda}\right)$ for FMS $\left(\alpha_{2}=1\right)$ (upper two curves) and Batchelor parametrization (lower two curves).

gitimate a choice as the latter. The local scaling exponent of $D_{B}(r)$ is given by

$$
\zeta(r)=2+\frac{2}{3} \frac{r^{2}}{L^{2}+r^{2}}-\frac{4}{3} \frac{r^{2}}{r_{d}^{\prime 2}+r^{2}},
$$

and is shown in Fig. 5(b). The apparent scaling correction is defined analogously to (26),

$$
\delta \zeta^{a p p, r}=\min _{r}[\zeta(r)]-m / 3 .
$$

For the same $\operatorname{Re}_{\lambda}=1500$ as above, $\delta \zeta^{a p p, r} \approx 0.0028$ is now much smaller than the corresponding value for the generalized FMS parametrization. This reflects the much better scaling properties of the Batchelor parametrization (compared to FMS; cf. Fig. 4), which we have extensively discussed in Secs. II and III. The $\operatorname{Re}_{\lambda}$ dependence of $\delta \zeta^{a p p, r}$ is displayed in Fig. 6. For large $L \gg r_{d}^{\prime}$ (i.e., for large $\operatorname{Re}_{\lambda}$ ) we obtain

$$
\delta \zeta^{a p p, r}=\sqrt{2} \frac{4}{3} \frac{r_{d}^{\prime}}{L} \propto \operatorname{Re}^{-3 / 4} \propto \operatorname{Re}_{\lambda}^{-3 / 2}
$$

This means that even the asymptotic dependences on $\operatorname{Re}_{\lambda}$ of $\delta \zeta^{a p p, p}$ (Batchelor) and $\delta \zeta^{a p p, p}$ (FMS) differ from each other. Let us reexamine these scaling properties after Fourier transforming (25) and (28).

\section{B. Fourier transforms}

Transforming the FMS-parametrization equation

$$
\begin{aligned}
D_{F M S}(r) \propto & \frac{\Gamma(-2 / 3)}{(5 / 3) r p_{d}^{5 / 3}} \\
& \times\left[\frac{5}{3} p_{d} r-\left(1+p_{d}^{2} r^{2}\right)^{5 / 6} \sin \left(\frac{5}{3} \arctan \left(p_{d} r\right)\right)\right] \\
& +\frac{\alpha_{2} \Gamma(-4 / 3) p_{L}^{2 / 3}}{(7 / 3) r p_{d}^{7 / 3}} \\
& \times\left[\frac{7}{3} p_{d} r-\left(1+p_{d}^{2} r^{2}\right)^{7 / 6} \sin \left(\frac{7}{3} \arctan \left(p_{d} r\right)\right)\right] .
\end{aligned}
$$

The local slope of Eq. (32) for $\operatorname{Re}_{\lambda}=1500, \alpha_{2}=1$, is also plotted in Fig. 5(a), to compare it with the slope of Eq. (25). Now $\delta \zeta^{a p p, r} \approx 0.11$ is even larger than $\delta \xi^{a p p, p}$ (both for FMS), which is clearly understandable from Fig. 4 because of the even worse scaling properties of the FMS parametrization in $r$ space (compared to $p$ space).

Surprisingly, we obtain for the $\operatorname{Re}_{\lambda}$ dependence of $\delta \xi^{a p p, r}$ (FMS) the asymptotic result

$$
\delta \zeta^{a p p, r}\left(\operatorname{Re}_{\lambda}\right) \propto \operatorname{Re}_{\lambda}^{-1 / 2}
$$

which differs considerably from (27). We conclude that, for the FMS parametrization, it matters a lot whether the apparent scaling corrections are determined in $r$ or in $p$ space. It is the $r$-space result (33) which has to be compared with the ( $r$-space) data of [1]. Note that the $\operatorname{Re}_{\lambda}$ dependence is still steeper than what is found experimentally $[1,5]$.

Finally, we calculate the spectrum corresponding to the generalized Batchelor parametrization (28). For $r \gg r_{d}^{\prime}$ we derive an analytical result in Appendix A, while in general the transformation is performed numerically employing a contour integration technique described in Appendix B. The result for the local $p$-space slope of the generalized Batchelor parametrization (28) is shown in Fig. 5(b). It shows the ultraviolet bottleneck energy pileup [30], which we had discussed in detail in Ref. [29]. In addition, the spectrum shows reduced spectral strength at the infrared end of the $p$-space ISR, i.e., a smaller local slope $\zeta(p)<2 / 3$. This effect can be interpreted as, so to say, an inverse bottleneck effect and can both formally and physically be interpreted along the same line of arguments as the bottleneck pileups discussed above and in [29]. Formally it reflects the sharp crossover from $r^{2 / 3}$ to $r^{4 / 3}$ scaling in the structure function. Physically $[20,29]$, the constant energy flux $T(p)$ $\sim p u(\boldsymbol{p}) \int d p_{1} d p_{2} u\left(\boldsymbol{p}_{1}\right) u\left(\boldsymbol{p}_{2}\right) \delta\left(\boldsymbol{p}+\boldsymbol{p}_{1}+\boldsymbol{p}_{2}\right)$ downscale now requires reduced spectral strength at the infrared end of the ISR, as the spectral strength is increased in the shear range. Correspondingly, there is also an energy pileup at the high$p$ end of the shear range, which may be a consequence of the constant helicity flux in this region [27]. It leads to a local slope $\zeta(p)>4 / 3$.

We see that, for the Batchelor parametrization, positive corrections $\delta \zeta(r)>0$ in $r$ space lead to negative corrections $\delta \zeta(p)<0$ in the $p$-space ISR. The apparent scaling corrections $\delta \zeta^{a p p, p}$ have to be defined as

$$
\delta \zeta_{2}^{a p p, p}=\max _{p_{L} \ll p \ll p_{d}}\left[\zeta_{2}(p)\right]-m / 3 .
$$


This definition is close in spirit to the experimental procedure, namely, looking for scaling exponents in the middle of the ISR. Also, it leads to $\delta \zeta_{2}^{a p p, p}\left(\operatorname{Re}_{\lambda} \rightarrow \infty\right)=0$ as required. The $\operatorname{Re}_{\lambda}$ dependence of $\delta \zeta^{a p p, p}$ is shown in Fig. 6. From the data we conclude that

$$
\delta \zeta^{a p p, p} \propto-\operatorname{Re}_{\lambda}^{-3 / 2}<0
$$

For $\operatorname{Re}_{\lambda}=1500$ we have $\delta \zeta^{a p p, p}=-0.0082$.

To summarize this section, we found that the crossovers at both ends of the ISR govern the $\operatorname{Re}_{\lambda}$ dependence of $\delta \zeta^{a p p}$. In the two examined cases either the exponent in the $\operatorname{Re}_{\lambda}$ dependence (FMS) or the overall sign (Batchelor) of $\delta \zeta$ changed when going from $r$ to $p$ space. We hope that our analysis leads to more detailed numerical or experimental studies of the ISR to shear crossover. This would help to clarify the question of the $\operatorname{Re}_{\lambda}$ dependence of scaling corrections.

\section{SUMMARY AND CONCLUSIONS}

Throughout the paper we have demonstrated that scaling properties in $r$ and in $p$ space can be quite different. In the infinite $\operatorname{Re}_{\lambda}$ limit these differences are of course irrelevant. Yet, as we demonstrated, for those $\operatorname{Re}_{\lambda}$ which can be achieved in experiments and even more so for the numerical ones, the finite size corrections are considerable and it is important to know what their influence is to be able to interpret the data correctly. The apparent scaling correction $\delta \zeta^{a p p}$ due to shear corrections even show asymptotically different $\operatorname{Re}_{\lambda}$ scaling behavior, depending on whether they are defined in $p$ or $r$ space.

Comparison of the size of the scaling ranges for given $\operatorname{Re}_{\lambda}$ between experiment [1] and our parametrizations makes us favor a Batchelor-type parametrization rather than a parametrization of FMS type. The latter, consisting of a power law in $p$ space with a large- $p$ exponential cutoff and a small$p$ arctan cutoff, does not exhibit any bottleneck energy pileups at the ends of the $p$-space ISR and leads to unrealistic short scaling ranges in the $r$-space structure function. In other words, combining all regimes discussed in this paper (VSR, ISR, shear range, and large- $r$ saturation range) we think that the $p$-space parametrization

$$
\begin{aligned}
E(p)= & \frac{2 E_{0} \epsilon^{2 / 3}}{\pi} \arctan \left[\left(\frac{p}{p_{L}}\right)^{11 / 3}\right] p^{-5 / 3}\left[1+\left(\frac{p}{p_{s}}\right)^{-2 / 3}\right] \\
& \times \exp \left(-p / p_{d}\right)
\end{aligned}
$$

with $p_{d} \geqslant p_{s} \geqslant p_{L}$ is less favorable than an $r$-space parametrization

$$
D(r)=\frac{\epsilon}{3 \nu} r^{2} \frac{r_{d}^{\prime 4 / 3}}{\left(r_{d}^{\prime 2}+r^{2}\right)^{2 / 3}} \frac{r_{s}^{-2 / 3}}{\left(r_{s}^{2}+r^{2}\right)^{-1 / 3}} \frac{L^{-4 / 3}}{\left(L^{2}+r^{2}\right)^{-2 / 3}}
$$

with $r_{d}^{\prime} \leqslant r_{s} \leqslant L$, which shows bottleneck effects in $p$ space. In many isotropic turbulence experiments $r_{s} \approx L$ and the shear range will be suppressed. If less isotropy is achieved in experiments, we may have, say, $r_{s} \approx L / 4$. In this case, the energy pileup due to large- $r$ saturation (Sec. II and Ref. [29]) and the spectral strength reduction due to shear effects will partly compensate each other at the infrared end of the $p$-space ISR. This leads to a smaller change of the local slope than predicted by Eq. (15). Also, experimentally only averaged slopes are accessible which partly smoothen the bottleneck bumps in the pointwise slopes. A better resolution may be necessary to further reveal the physics of the crossover regions which might well be a key in understanding scaling corrections.

\section{ACKNOWLEDGMENTS}

We thank A. Esser, G. Falkovich, S. Grossmann, L. Kadanoff, and R. Kerr for helpful suggestions, V. Yakhot for supplying us with the results of his work prior to publication, and M. Nelkin for bringing the history of Eq. (3) to our attention. D.L. heartily thanks G. Falkovich for his hospitality during his stay at the Weizmann Institute of Science, Rehovot, where part of the work was done, and kindly acknowledges support by the Einstein Foundation, by a NATO grant through the Deutsche Akademische Austauschdienst (DAAD), and by the DOE. A.M.-G. was supported by the Natural Sciences and Engineering Research Council of Canada.

\section{APPENDIX A: ANALYTICAL BATCHELOR-TYPE SHEAR SPECTRUM}

We can analytically perform the transformation of the Batchelor-parametrization equation (28) under the assumption that $r \gg r_{d}^{\prime}$, so that

$$
D(r) \approx \frac{\epsilon}{3 \nu}\left(\frac{r}{r_{s}}\right)^{2 / 3} \frac{1}{\left(r_{s}^{2}+r^{2}\right)^{-1 / 3}} .
$$

With this approximation we get

$$
\begin{aligned}
E(p)= & -\frac{1}{2 \pi} \int_{0}^{\infty} p r D^{(2)}(r) \sin (p r) d r \\
= & \frac{p}{2 \pi} \frac{\epsilon}{3 \nu r_{s}^{2 / 3}} \frac{d}{d p}\left(r_{s}^{2}-\frac{d^{2}}{d p^{2}}\right) \\
& \times \int_{0}^{\infty} r^{2 / 3}\left(r_{s}^{2}+r^{2}\right)^{-2 / 3} \cos (p r) d r .
\end{aligned}
$$

The integral in Eq. (A2) can be solved [44] to give

$$
\begin{aligned}
\int_{0}^{\infty} r^{2 / 3}\left(r_{s}^{2}+r^{2}\right)^{-2 / 3} \cos (p r) d r \\
=-3 r_{s}^{1 / 3} \frac{\Gamma(5 / 6)^{2}}{\Gamma(2 / 3)}{ }_{1} F_{2}\left(\frac{5}{6} ; \frac{1}{2}, \frac{7}{6} ; \frac{\tilde{p}^{2}}{4}\right) \\
\quad+\frac{\sqrt{3}}{2} \Gamma(1 / 3) p_{1}^{-1 / 3} F_{2}\left(\frac{2}{3} ; \frac{1}{3}, \frac{5}{6} ; \frac{\tilde{p}^{2}}{4}\right),
\end{aligned}
$$

where $\tilde{p}=p r_{s}$. Reinserting this result into Eq. (A2) we derive the following, rather clumsy expression: 


$$
\begin{aligned}
E(p)= & \frac{\epsilon r_{s}^{5 / 3}}{3 \nu}\left(\frac{15}{14 \pi} \frac{\Gamma(5 / 6)^{2}}{\Gamma(2 / 3)} \tilde{p}^{2}\left[\frac{11}{13}{ }_{1} F_{2}\left(\frac{17}{6} ; \frac{5}{2}, \frac{19}{6} ; \frac{\tilde{p}^{2}}{4}\right)-{ }_{1} F_{2}\left(\frac{11}{6} ; \frac{3}{2}, \frac{13}{6} ; \frac{\tilde{p}^{2}}{4}\right)+\frac{187}{3705}{ }_{1} F_{2}\left(\frac{23}{6} ; \frac{7}{2}, \frac{25}{6} ; \frac{\tilde{p}^{2}}{4}\right) \tilde{p}^{2}\right]\right. \\
& +\frac{\sqrt{3}}{4 \pi} \Gamma(1 / 3) \tilde{p}^{-7 / 3}\left\{\frac{28}{27}{ }_{1} F_{2}\left(\frac{2}{3} ; \frac{1}{3}, \frac{5}{6} ; \frac{\tilde{p}^{2}}{4}\right)-\left[\frac{1}{3}{ }_{1} F_{2}\left(\frac{2}{3} ; \frac{1}{3}, \frac{5}{6} ; \frac{\tilde{p}^{2}}{4}\right)+\frac{2}{5}{ }_{1} F_{2}\left(\frac{5}{3} ; \frac{4}{3}, \frac{11}{6} ; \frac{\tilde{p}^{2}}{4}\right)\right] \tilde{p}^{2}\right. \\
& \left.\left.+\left[\frac{6}{5}{ }_{1} F_{2}\left(\frac{5}{3} ; \frac{4}{3}, \frac{11}{6} ; \frac{\tilde{p}^{2}}{4}\right)-\frac{9}{11}{ }_{1} F_{2}\left(\frac{8}{3} ; \frac{7}{3}, \frac{17}{6} ; \frac{\tilde{p}^{2}}{4}\right)\right] \tilde{p}^{4}-\frac{108}{1309}{ }_{1} F_{2}\left(\frac{11}{3} ; \frac{10}{3}, \frac{23}{6} ; \frac{\tilde{p}^{2}}{4}\right) \tilde{p}^{6}\right\}\right) .
\end{aligned}
$$

For small $p$ the local scaling exponent of $E(p)$ is $4 / 3$, for large $p$ it is $2 / 3$, but the transition from one range to the other is nonmonotonous. This is reflected in the right part of Fig. 5(b). In the left part of that figure, the ultraviolet bottleneck energy pileup can be seen in addition, which is not included in Eqs. (A1) and (A4).

\section{APPENDIX B: CONTOUR INTEGRATION FOR OSCILLATING INTEGRANDS}

The numerical Fourier transformation (7) of (28) cannot straightforwardly be performed, as the integrand is strongly oscillating and not exponentially damped. To cope with this problem, we employ contour integration techniques [49]. Plugging (28) into (7) we obtain after some algebra

$$
E(p)=-\frac{\epsilon}{3 \nu} r_{d}^{\prime 4 / 3} r_{s}^{-2 / 3} \frac{p}{4 \pi} \frac{d^{3}}{d p^{3}} \int_{-\infty}^{\infty} \frac{\left(r_{s}^{2}+r^{2}\right)^{1 / 3} \exp (i p r)}{\left(r_{d}^{\prime 2}+r^{2}\right)^{2 / 3}} d r
$$

The integral has singularities or zeros at $\pm i r_{d}^{\prime}$ and $\pm i r_{s}$. Taking the correct branch cuts and performing the corresponding contour integration in the upper half-plane, we obtain

$$
\begin{aligned}
\int_{-\infty}^{\infty} & \frac{\left(r_{s}^{2}+r^{2}\right)^{1 / 3} \exp (i p r)}{\left(r_{d}^{\prime 2}+r^{2}\right)^{2 / 3}} d r \\
& =-\sqrt{3} \int_{r_{d}^{\prime}}^{\infty} \frac{\left|z^{2}-r_{s}^{2}\right|^{1 / 3} \exp (-p z)}{\left(z^{2}-r_{d}^{\prime 2}\right)^{2 / 3}} d z
\end{aligned}
$$

or

$$
E(p)=\frac{\epsilon}{3 \nu} r_{d}^{\prime 4 / 3} r_{s}^{-2 / 3} \frac{\sqrt{3} p}{4 \pi} \int_{r_{d^{\prime}}}^{\infty} \frac{z^{3}\left|z^{2}-r_{s}^{2}\right|^{1 / 3} \exp (-p z)}{\left(z^{2}-r_{d}^{\prime 2}\right)^{2 / 3}} d z
$$

which can now be straightforwardly integrated. Our numerical result is displayed in Fig. 5(b).
[1] F. Anselmet, Y. Gagne, E. J. Hopfinger, and R. Antonia, J. Fluid Mech. 140, 63 (1984); B. Castaing, Y. Gagne, and E. J. Hopfinger, Physica D 46, 177 (1990); B. Castaing, Y. Gagne, and M. Marchand, ibid. 68, 387 (1993); B. Chabaud, A. Naert, J. Peinke, F. Chilla, B. Castaing, and B. Hebral, Phys. Rev. Lett. 73, 3227 (1994).

[2] A. A. Praskovsky, Phys. Fluids A 4, 2589 (1992).

[3] A. Praskovsky and S. Oncley, Phys. Fluids A 6, 2886 (1994).

[4] G. Zocchi, P. Tabeling, J. Maurer, and H. Willaime, Phys. Rev. E 50, 3639 (1994); J. Maurer, P. Tabeling, and G. Zocchi, Europhys. Lett. 26, 31 (1994).

[5] F. Belin, P. Tabeling, and H. Willaime, Physica D (to be published).

[6] S. G. Saddoughi and S. V. Veeravalli, J. Fluid Mech. 268, 333 (1994).

[7] S. G. Saddoughi (unpublished).

[8] K. Sreenivasan, Proc. R. Soc. London A 434, 165 (1991).

[9] R. Kerr, J. Fluid Mech. 211, 309 (1990); A. Vincent and M. Meneguzzi, ibid. 225, 1 (1991).

[10] Z. S. She et al., Phys. Rev. Lett. 70, 3251 (1993).

[11] V. Borue and S. A. Orszag, Phys. Rev. E 51, R856 (1995).

[12] R. H. Kraichnan, J. Fluid Mech. 5, 497 (1959); D. C. Leslie, Developments in the Theory of Turbulence (Clarendon Press, Oxford, 1973); H. W. Wyld, Ann. Phys. (N.Y.) 14, 1961 (1961).
[13] V. S. L’vov and I. Procaccia, Phys. Rev. E 49, 4044 (1994).

[14] V. S. L'vov and I. Procaccia, Phys. Rev. Lett. 74, 2690 (1995).

[15] A. N. Kolmogorov, C. R. Acad. Sci. USSR 30, 299 (1941); L. Onsager, Phys. Rev. 68, 286 (1945); A. M. Obukhov, Izv. Akad. Nauk SSSR Ser. Geog. Geofiz. 13, 58 (1949); W. Heisenberg, Z. Phys. 124, 628 (1948); C. F. von Weizsäcker, Z. Phys. 124, 614 (1948).

[16] L. D. Landau and E. M. Lifshitz, Fluid Mechanics (Pergamon Press, Oxford, 1987).

[17] A. N. Kolmogorov, J. Fluid Mech. 13, 82 (1962); U. Frisch, P. L. Sulem, and M. Nelkin, ibid. 87, 719 (1978); G. Parisi and U. Frisch, in Turbulence and Predictability of Geophysical Fluid Dynamics, edited by M. Ghil, R. Benzi, and G. Parisi (North-Holland, Amsterdam, 1985), p. 84; C. Meneveau and K. R. Sreenivasan, Phys. Rev. Lett. 59, 1424 (1987); J. Fluid Mech. 224, 429 (1991); Z. S. She and E. Leveque, Phys. Rev. Lett. 72, 336 (1994).

[18] M. Nelkin, Phys. Fluids 24, 556 (1981).

[19] S. Grossmann and D. Lohse, Phys. Fluids 6, 611 (1994).

[20] S. Grossmann and D. Lohse, Phys. Rev. E 50, 2784 (1994).

[21] J. Eggers and S. Grossmann, Phys. Fluids A 3, 1958 (1991).

[22] S. Grossmann, D. Lohse, and A. Reeh, in Dynamical Systems and Chaos, Vol. 2: Physics, edited by Y. Aizawa, S. Saito, and K. Shiraiwa (World Scientific, Singapore, 1995), pp. 209-217. 
[23] S. Grossmann, D. Lohse, V. L'vov, and I. Procaccia, Phys. Rev. Lett. 73, 432 (1994).

[24] J. L. Lumley, Phys. Fluids 10, 855 (1967); H. Tennekes and J. L. Lumley, A First Course in Turbulence (The MIT Press, Cambridge, MA, 1972).

[25] E. A. Kuznetsov and V. S. L'vov, Physica D 2, 203 (1981); G. E. Falkovich, in Nonlinear Waves and Weak Turbulence, edited by N. Fitzmaurice, D. Gurarie, F. McCaughan, and W. A. Woyczynski (Birkhäuser, Boston, 1993), p. 19.

[26] V. Yakhot, Phys. Rev. E 49, 2887 (1994).

[27] D. Lohse, Phys. Lett. A 196, 70 (1994).

[28] A. S. Monin and A. M. Yaglom, Statistical Fluid Mechanics (The MIT Press, Cambridge, MA, 1975).

[29] D. Lohse and A. Müller-Groeling, Phys. Rev. Lett. 74, 1747 (1995).

[30] G. Falkovich, Phys. Fluids 6, 1411 (1994).

[31] M. Nelkin, Adv. Phys. 43, 143 (1994).

[32] C. Foias, O. Manley, and L. Sirovich, Phys. Fluids A 2, 464 (1990).

[33] U. Frisch and R. Morf, Phys. Rev. A 23, 2673 (1981); M. E. Brachet et al., J. Fluid Mech. 130, 411 (1983).

[34] G. K. Batchelor, Proc. Camb. Philos. Soc. 47, 359 (1951).

[35] H. Effinger and S. Grossmann, Z. Phys. B 66, 289 (1987).

[36] L. Sirovich, L. Smith, and V. Yakhot, Phys. Rev. Lett. 72, 344 (1994); 74, 1492(E) (1995).
[37] I. Procaccia et al., Phys. Rev. A 44, 8091 (1991).

[38] Z. S. She and E. Jackson, Phys. Fluids A 5, 1526 (1993).

[39] R. Benzi, S. Ciliberto, C. Baudet, and G. R. Chavarria, Physica $\mathrm{D}$ (to be published).

[40] G. Stolovitzky, K. R. Sreenivasan, and A. Juneja, Phys. Rev. E 48, R3217 (1993).

[41] K. R. Sreenivasan, Phys. Fluids 7, 2778 (1995).

[42] M. Abramowitz and I. A. Stegun, Handbook of Mathematical Functions (Dover, New York, 1970).

[43] J. R. Herring, in Unstable and Turbulent Motion of Fluid, edited by S. Kida (World Scientific, Singapore, 1993).

[44] A. P. Prudnikov, Y. A. Brychkov, and O. I. Marichev, Integrals and Series (Gordon and Breach, New York, 1990).

[45] J. R. Herring, D. Schertzer, M. Lesieur, G. R. Newman, J. P. Chollet, and M. Larcheveque, J. Fluid Mech. 124, 411 (1982); J. Qian, Phys. Fluids 27, 2229 (1984); V. I. Tatarskii, M. M. Dubovikov, A. A. Praskovsky, and M. Y. Karyakin, J. Fluid Mech. 238, 683 (1992).

[46] S. A. Orszag, in Fluid Dynamics, Les Houches Summer School, edited by R. Balian and J. L. Peube (Gordon and Breach, New York, 1977), pp. 237-374.

[47] K. R. Sreenivasan, Phys. Fluids 27, 1048 (1984).

[48] D. Lohse, Phys. Rev. Lett. 73, 3223 (1994).

[49] G. N. Watson, A Treatise on the Theory of Bessel Functions (Cambridge University Press, Cambridge, 1922). 